\title{
The Effect of Dimethyl Fumarate on Cerebral Gray Matter Atrophy in Multiple Sclerosis
}

Sheena L. Dupuy · Shahamat Tauhid · Shelley Hurwitz •

Renxin Chu $\cdot$ Fawad Yousuf · Rohit Bakshi (D)

Received: September 12, 2016 / Published online: October 15, 2016

(c) The Author(s) 2016. This article is published with open access at Springerlink.com

\section{ABSTRACT}

Introduction: The objective of this pilot study was to compare cerebral gray matter (GM) atrophy over 1 year in patients starting dimethyl fumarate (DMF) for multiple sclerosis (MS) to that of patients on no disease-modifying treatment (noDMT). DMF is an established therapy for relapsing-remitting (RR) MS.

Methods: We retrospectively analyzed 20 patients with RRMS at the start of DMF [age (mean \pm SD) $\quad 46.1 \pm 10.2$ years, Expanded Disability Status Scale (EDSS) score $1.1 \pm 1.2$,

Enhanced content To view enhanced content for this article go to www.medengine.com/Redeem/ 1D07F06062DBE32A.

S. L. Dupuy · S. Tauhid · R. Chu · F. Yousuf Department of Neurology, Laboratory for Neuroimaging Research, Partners MS Center, Harvard Medical School, Brigham and Women's Hospital, Boston, MA, USA

S. Hurwitz

Department of Medicine, Harvard Medical School, Brigham and Women's Hospital, Boston, MA, USA

R. Bakshi ( $\square)$

Departments of Neurology and Radiology, Laboratory for Neuroimaging Research, Partners MS Center, Harvard Medical School, Brigham and

Women's Hospital, Boston, MA, USA

e-mail: rbakshi@post.harvard.edu timed 25-foot walk (T25FW) $4.6 \pm 0.8 \mathrm{~s}$ ] and eight patients on noDMT (age $42.5 \pm 6.6$ years, EDSS $1.7 \pm 1.1$, T25FW $4.4 \pm 0.6 \mathrm{~s}$ ). Baseline and 1-year 3D T1-weighted 3T MRI was processed with automated pipelines (SIENA, FSL-FIRST) to assess percentage whole brain volume change (PBVC) and deep GM (DGM) atrophy. Group differences were assessed by analysis of covariance, with time between MRI scans as a covariate.

Results: Over 1 year, the DMF group showed a lower rate of whole brain atrophy than the noDMT group (PBVC: $-0.37 \pm 0.49 \%$ vs. $-1.04 \pm 0.67 \%, p=0.005)$. The DMF group also had less change in putamen volume $(-0.06 \pm 0.22$ vs. $-0.32 \pm 0.28 \mathrm{ml}, p=0.02)$. There were no significant on-study differences between groups in caudate, globus pallidus, thalamus, total DGM volume, T2 lesion volume, EDSS, or T25FW (all $p>0.20$ ).

Conclusions: These results suggest a treatment effect of DMF on GM atrophy appearing at 1 year after starting therapy. However, due to the retrospective study design and sample size, these findings should be considered preliminary, and require confirmation in future investigations.

Funding: Biogen. 
Keywords: Cerebral gray matter atrophy; Dimethyl fumarate; Multiple sclerosis

\section{INTRODUCTION}

Conventional brain MRI measures of multiple sclerosis (MS)-related disease activity, such as the number of gadolinium (Gad)-enhancing lesions and new or enlarging T2-weighted lesions, are standard supportive outcome measures in clinical trials of disease-modifying therapies (DMTs) [1-4]; however, additional measures have been shown to provide complementary information on the efficacy of DMTs $[1,5,6]$. Brain atrophy has become increasingly important due to its core role in the pathophysiology of MS, correlations with clinical dysfunction, and the technological post-processing advances allowing a continuing increase in the sophistication of MRI quantification methods [1, 5-19]. In addition, the use of higher-field (i.e., 3T) MRI scanners provides the potential for increased accuracy and reliability in the assessment of lesions and atrophy $[9,10,20]$. Brain atrophy was once thought to be predominantly the result of white matter (WM) volume loss, but it is now widely accepted that gray matter (GM) bears the brunt of such atrophy [13]. Furthermore, several studies have indicated that GM atrophy may be a more reliable marker of treatment effects than WM atrophy, as WM volume is prone to fluctuations [21]; this is partly on the basis of pseudoatrophy in the first few months after the initiation of DMTs [22, 23], or transient increases in WM volume due to inflammation [24]. GM atrophy occurs early in the MS disease course, is related to immunologic changes, physical disability, cognitive dysfunction, depression, and quality of life, and can predict long-term clinical changes $[1,5,7,8,10,11,13,15,17,18,25-33]$. Thus, assessing therapeutic effects on GM atrophy is an attractive strategy for further exploration [12].

Phase III MS clinical trials have shown that several DMTs partially, but significantly, reduce the rate of whole brain atrophy when compared to placebo treatment $[6,13,34-44]$; however, assessments of such effects on cerebral GM atrophy are largely limited to smaller post hoc or phase IV studies [12, 18, 19, 23, 45-47].

Dimethyl fumarate (DMF) is an orally administered agent approved for the treatment of relapsing-remitting (RR) MS. Its proposed mechanism of action involves the activation of the nuclear factor (erythroid-derived 2)-like 2 (Nrf2) pathway and Nrf2 independent pathways to increase resistance against oxidative stress, potentially providing both anti-inflammatory and neuroprotective effects [48, 49]. DMF has been shown to significantly reduce relapse rates, progression of physical disability, and the accumulation of brain MRI lesions in patients with RRMS when compared to placebo [37, 38, 50-54]. The pivotal phase III trials have also shown that DMF therapy significantly reduces the rate of whole brain volume loss compared to placebo [37, 38]. However, no published study to date has assessed the impact of DMF on GM damage, such as the progression of GM atrophy. The objective of this pilot study was to compare the 1-year rate of cerebral GM atrophy in patients with RRMS after the start of DMF, as compared to untreated patients, in a "real-world" clinical care setting.

\section{METHODS}

\section{Subjects}

Baseline demographic and clinical characteristics of all subjects are presented in 
Table 1. We retrospectively analyzed 20 consecutive patients with RRMS who were newly starting oral DMF therapy (120 mg twice a day for 1 week, then $240 \mathrm{mg}$ twice a day) and eight patients (six RRMS, two clinically isolated syndrome) on no disease-modifying therapy (noDMT) for 1 year. All subjects were identified by chart review using the following inclusion criteria: age 18-60 years, Expanded Disability Status Scale (EDSS) score of $0-5$ at baseline, no corticosteroid use within 30 days of MRI, and the availability of baseline and 1-year follow-up brain 3T MRI. Prior to starting DMF, patients had been previously treated with the following DMTs: glatiramer acetate (GA; $n=8)$, intramuscular interferon beta-1a (IFN $\beta-1 \mathrm{a}$, once a week; $n=7)$, subcutaneous interferon beta-1a (thrice weekly; $n=4$ ), interferon beta- $1 \mathrm{~b}$ $(n=2)$, and natalizumab $(n=2)$. Patients in the noDMT group had no prior history of exposure to any DMT. All patients underwent clinical evaluations, including EDSS scoring and timed 25-foot walk (T25FW), within 3 months of MRI by an MS specialist neurologist at our institution. All procedures followed were in accordance with the ethical standards of the responsible committee on human experimentation (institutional and national) and with the Helsinki Declaration of 1964, as revised in 2013. Informed consent was not required due to the retrospective nature of the study.

Table 1 Baseline demographic, clinical, and MRI data

\begin{tabular}{|c|c|c|c|}
\hline & DMF & noDMT & $p$ value \\
\hline Number of patients with multiple sclerosis & 20 & 8 & - \\
\hline Age (years) & $46.1 \pm 10.2(26.9-60.7)$ & $42.5 \pm 6.6(30.7-50.8)$ & $0.47^{*}$ \\
\hline Women, no. (\%) & $13(65 \%)$ & $8(100 \%)$ & $0.07^{\dagger}$ \\
\hline Disease duration (years)* & $12.4 \pm 8.0(1.3-35.7)$ & $6.7 \pm 6.8(0.2-17.0)$ & $0.15^{*}$ \\
\hline Expanded Disability Status Scale score & $1.1 \pm 1.2(0-3.5)$ & $1.7 \pm 1.1(0-3.0)$ & $0.22^{*}$ \\
\hline Timed 25-foot walk (s) & $4.6 \pm 0.8(3.3-6.7)$ & $4.4 \pm 0.6(3.5-5.2)$ & $0.68^{*}$ \\
\hline Time on DMF vs. baseline MRI (months) ${ }^{* *}$ & $2.0 \pm 2.8(-2$ to 6$)$ & - & - \\
\hline Thalamus volume $(\mathrm{ml})$ & $15.2 \pm 1.7(13.0-18.3)$ & $14.5 \pm 0.8(13.4-15.6)$ & $0.33^{*}$ \\
\hline Caudate volume $(\mathrm{ml})$ & $6.4 \pm 0.8(5.1-8.0)$ & $6.5 \pm 1.0(5.0-8.1)$ & $0.90^{*}$ \\
\hline Putamen volume $(\mathrm{ml})$ & $9.4 \pm 1.0(7.6-11.6)$ & $9.4 \pm 1.2(7.9-11.1)$ & $0.98^{*}$ \\
\hline Globus pallidus volume $(\mathrm{ml})$ & $3.5 \pm 0.4(2.8-4.4)$ & $3.3 \pm 0.3(2.9-3.7)$ & $0.28^{*}$ \\
\hline Total DGM volume $(\mathrm{ml})$ & $34.6 \pm 3.3(29.6-42.1)$ & $33.7 \pm 3.0(29.7-38.3)$ & $0.53^{*}$ \\
\hline Cerebral T2 lesion volume (ml) & $8.6 \pm 11.6(0.4-46.5)$ & $2.7 \pm 1.7(0.6-6.2)$ & $0.28^{\ddagger}$ \\
\hline Cerebral \# of gadolinium enhancing lesions & $0.9 \pm 1.3(0-5)$ & $0.3 \pm 0.5(0-1)$ & $0.35^{\S}$ \\
\hline
\end{tabular}

Data are shown as mean \pm standard deviation (range), except as otherwise indicated; * years from first symptoms; $D M F$ dimethyl fumarate; noDMT no disease-modifying therapy; total cerebral deep gray matter $(D G M)=$ thalamus + caudate + putamen + globus pallidus; ${ }^{* *}$ negative number indicates that therapy was started after baseline MRI; ${ }^{\dagger}$ Fisher's exact test; ${ }^{\ddagger}$ Exact Wilcoxon test; ${ }^{\S}$ Exact Jonckheere-Terpstra test 


\section{MRI Acquisition}

All subjects underwent 3T brain MRI using the same scanner (3T Skyra; Siemens Medical Solutions, Malvern, PA, USA) and similar acquisition protocol. We chose 3T for this study based on the higher diagnostic sensitivity it has shown in the detection of brain lesions and atrophy in MS [9, 10, 20]. The relevant images included (1) sagittal 3D T1-weighted magnetization-prepared rapid acquisition gradient echo (MPRAGE) images (TR/TE: $2300 / 2.96 \mathrm{~ms}$ for all except one patient with TR/TE: $1800 / 2.84 \mathrm{~ms}$; voxel size: $1 \times 1 \times 1 \mathrm{~mm}$ ) and (2) axial 2D T2-weighted fluid-attenuated inversion-recovery (FLAIR) images (TR/TE: 9000/81 ms; voxel size: $0.6875 \times 0.6875 \times 3 \mathrm{~mm}$ for all except one patient with voxel size: $0.4297 \times$ $0.4297 \times 3 \mathrm{~mm}$ ). In addition, axial 2D T1-weighted spin-echo post-contrast images were acquired 5-7 min after a single dose of intravenous Gad (TR/TE: 474-916/88-200 ms; voxel size: $0.4297 \times 0.4297 \times 3 \mathrm{~mm}$ for all except a patient with $0.8594 \times 0.8594 \times$ $3 \mathrm{~mm}$ ). For the DMF group, baseline MRI was conducted within (mean $\pm \mathrm{SD}$ ) $2.0 \pm 2.8$ months (range 6 months prior to 2 months post) of the start of DMF. Follow-up MRI for all subjects was obtained at a similar interval between groups within a range of 9-15 months after baseline MRI (DMF group: $12.2 \pm 1.2$ months; noDMT group: $11.8 \pm 2.1$ months; $p=0.59$ ).

\section{MRI Analysis}

\section{Pre-processing}

All image pre-processing was performed using Jim software (version 7.0; Xinapse Systems Ltd., West Bergholt, UK, http://www.xinapse.com/). The raw sagittal images did not yield adequate segmentation, particularly of the intracranial volume cavity (data not shown). Based on optimization work, all original DICOM images were first converted to a Neuroimaging Informatics Technology Initiative (NIfTI) format, and their native sagittal orientation was converted to axial. Axial slices were then extracted from each scan starting at the first slice showing the top of the head and continuing to the foramen magnum. This provided whole brain coverage in all subjects.

\section{Global Cerebral Atrophy}

The MPRAGE images were applied to an automated pipeline, the Structural Image Evaluation using Normalization of Atrophy (SIENA v. 5.0; Analysis Group, Oxford, UK, http://fsl.fmrib.ox.ac.uk), to assess the intra-subject percentage whole brain volume change (PBVC) between baseline and follow-up scans (Fig. 1). The use of SIENA to assess longitudinal whole brain atrophy is well established [14, 27, 38, 55]. To assess the reliability of this method with our acquisitions, three randomly chosen patients with MS and three healthy controls underwent an MPRAGE scan followed by a re-scan on the same day. Thus, two scans were acquired from each subject, where the subject was removed from the scanner for a few minutes between scans, and was repositioned and re-scanned by the MRI technologist. The MPRAGE acquisitions were performed with the same scanner platform and acquisition protocol as that employed in the present study. In these six scan-re-scan subjects, the PBVC was $-0.080 \pm 0.26 \%$, indicating high reliability.

\section{Cerebral Deep Gray Matter (DGM) Atrophy}

MPRAGE images were applied to an automated model-based segmentation tool, the Oxford Centre for Functional MRI of the Brain 


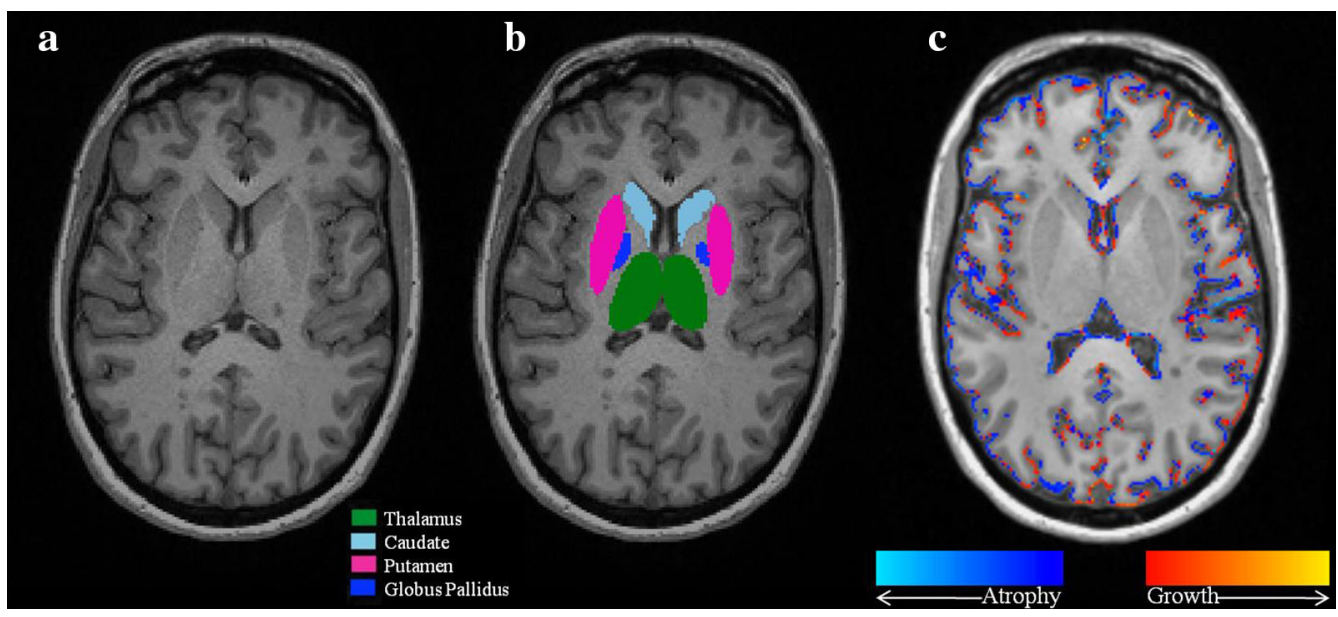

Fig. 1 Brain volume measurement techniques: regional and global metrics. A raw 3D T1-weighted magnetizationprepared rapid acquisition gradient echo (MPRAGE) image that has been re-sampled to axial (a) and an example of an FSL/FIRST [FMRIB (Oxford Centre for Functional MRI of the Brain) Software Library Integrated Registration and Segmentation Tool] deep gray matter segmentation (b) of the same MRI slice. In the present study, we utilized the FSL/FIRST outputs to assess the volume of the thalamus, caudate, putamen, and globus pallidus (and their sum $=$ total deep gray matter). In addition, we employed SIENA (Structural Image Evaluation using Normalization of Atrophy) to create a difference map in each subject to assess the within-subject

Software Library Integrated Registration and Segmentation Tool (FSL-FIRST, version 5.0; Analysis Group, Oxford, UK, http://fsl.fmrib. ox.ac.uk), to determine whole-structure volume of the putamen, caudate, globus pallidus, and thalamus. The total DGM was the sum of these four structures (Fig. 1). The use of FSL-FIRST to determine the volume of sub-cortical structures is well established $[9,26,56]$. Our previous experience with this method shows high scan-re-scan reliability, with an average intraclass correlation coefficient of 0.99 [9].

\section{Lesion Analysis}

Whole brain T2 hyperintense lesion volume (T2LV) analysis was performed using a change in whole brain volume from baseline to 1-year follow-up. One anatomic slice from the SIENA output is shown (c) for a subject. The colors on the image indicate the brain parenchymal change between baseline and follow-up, based on shifts of the brain boundary. Lighter blue colors indicate brain tissue volume loss over time (atrophy), whereas yellow colors indicate tissue volume increase. In this case, the patient experienced whole brain atrophy-percentage whole brain volume change of $-1.69 \%$. The images are from a 31-year-old woman with multiple sclerosis from the no disease-modifying therapy group, disease duration 2.4 years, baseline Expanded Disability Status Scale score 2, and baseline timed 25-foot walk $5.2 \mathrm{~s}$

semi-automated method based on expert tracing from the FLAIR images using Jim software (version 7; Xinapse Systems Ltd., West Bergholt, UK; http://www.xinapse.com), as previously described [20]. The number of cerebral Gad-enhancing lesions was determined from axial 2D T1-weighted post-Gad images by the same observer. Our methods for quantifying lesions have been shown to be highly reliable, as detailed previously [57].

All analysis was conducted in a blinded manner; researchers were unaware of clinical and demographic characteristics. Brain atrophy analysis was conducted by one observer (RC). Lesion analysis was conducted by a single observer (SD) and confirmed by a senior observer (ST). 


\section{Statistical Analysis}

Baseline demographic and clinical comparisons were conducted using exact Wilcoxon and Jonckheere-Terpstra tests. Cerebral GM atrophy measures were assessed by analysis of covariance with time between scans as a covariate. Lesion comparisons were assessed using Wilcoxon rank sum, Fisher's exact, and van Elteren tests (when adjusting for time between scans). Correlations were assessed using Spearman correlations. A value of $p<0.05$ was considered statistically significant; $p \geq 0.05$ but $<0.10$ was considered a trend toward significance.

\section{RESULTS}

\section{Baseline Demographic, Clinical, and MRI Comparisons}

No significant differences were detected between the DMF and noDMT groups in terms of baseline age, EDSS score, or T25FW (all $p>0.20$ ). The DMF and noDMT groups did not differ in terms of baseline T2LV, the number of Gad-enhancing lesions, or DGM volumes (all $p>0.25$ ) (Table 1).

\section{On-Study Change in Global Cerebral Atrophy}

In comparisons of the change in whole brain volume from baseline to follow-up, the DMF group showed less atrophy, with a lower PBVC, than the noDMT group $(-0.37 \pm 0.49 \%$ vs. $-1.04 \pm 0.67 \%, p=0.02$; when taking into account the time between baseline and follow-up MRI scans: $p=0.005$ ) (Table 2; Fig. 2).
Table 2 Brain volume change on-study: global and regional deep gray matter

\begin{tabular}{llll}
\hline & DMF & noDMT & $\boldsymbol{p}$ value \\
\hline Thalamus & $-0.10 \pm 0.28$ & $-0.04 \pm 0.34$ & 0.50 \\
Caudate & $-0.08 \pm 0.12$ & $-0.01 \pm 0.15$ & 0.28 \\
Putamen & $-0.06 \pm 0.22$ & $-0.32 \pm 0.28$ & $0.02^{*}$ \\
Globus pallidus & $-0.04 \pm 0.11$ & $-0.04 \pm 0.10$ & 1.00 \\
Total DGM & $-0.28 \pm 0.42$ & $-0.41 \pm 0.80$ & 0.69 \\
PBVC & $-0.37 \pm 0.49$ & $-1.04 \pm 0.67$ & $0.005^{*}$ \\
\hline
\end{tabular}

Data represent mean \pm standard deviation difference between baseline and 1-year follow-up in $\mathrm{ml}$ for all variables except for percent whole brain volume change (PBVC), which shows \%; DMF dimethyl fumarate; noDMT no disease-modifying therapy; total cerebral deep gray matter $(\mathrm{DGM})=$ thalamus + caudate + putamen + globus pallidus; $p$ values shown are with time between scans as a covariate; ${ }^{*} p<0.05$

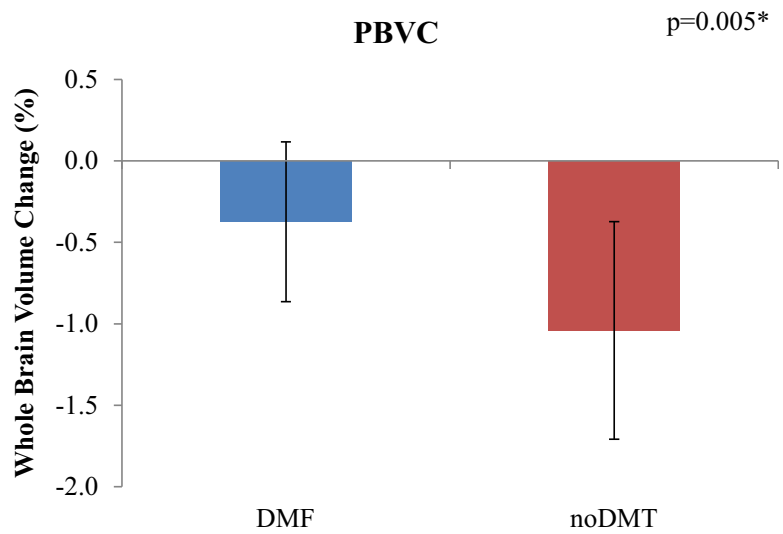

Fig. 2 Brain volume change on-study: whole brain atrophy. Percentage whole brain volume changes (PBVC) from baseline to follow-up were assessed in patients with multiple sclerosis on dimethyl fumarate (DMF) therapy vs. patients on no disease-modifying therapy (noDMT). Means and standard deviation bars are shown. On average, the DMT group had a $64.4 \%$ lower rate of whole brain atrophy than the noDMT group $(p=0.02 ; p=0.005$, adjusted for MRI interval). ${ }^{*} p<0.05$ 


\section{On-Study Change in DGM Atrophy}

In comparisons of on-study DGM volume changes, the DMF group showed significantly less atrophy, with smaller putamen volume changes, than the noDMT group $(-0.06 \pm 0.22$ vs. $-0.32 \pm 0.28 \mathrm{ml}, p=0.04)$. There were no significant on-study differences between groups in caudate, globus pallidus, thalamus, or total DGM volumes (all $p>0.35$ ) (Table 2; Fig. 3).
Similar results were found when adjusting for the time between baseline and follow-up MRI scans (putamen $p=0.02$; other volumes $p>0.25$ ) (Table 2; Fig. 3).

\section{On-Study Change in Lesions}

In terms of on-study T2LV and Gad count change, no significant differences were found between the two groups (both $p>0.40$ ). Similar
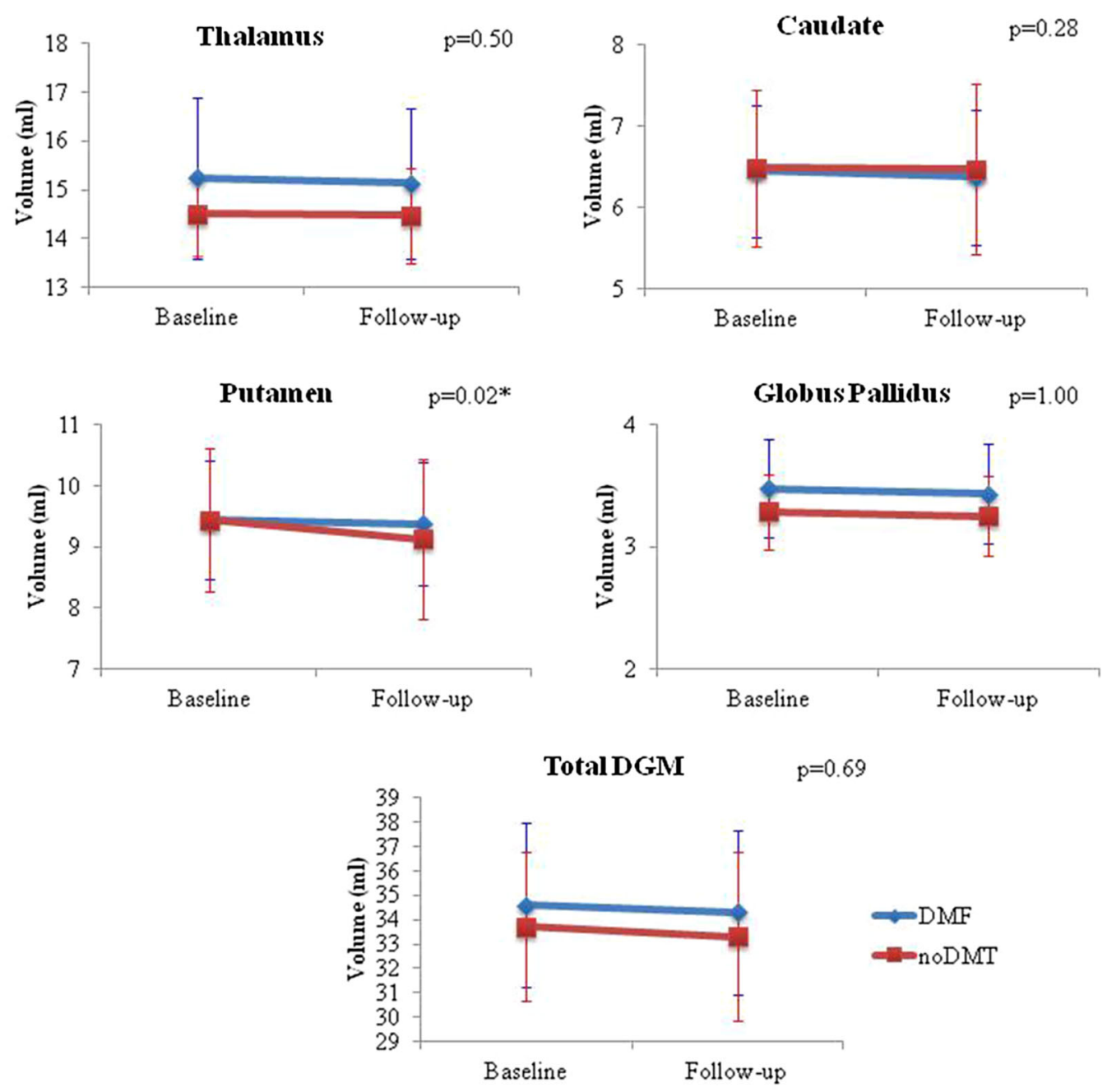

Fig. 3 Brain volume change on-study: regional (deep gray matter) atrophy. Changes in cerebral deep gray matter (DGM) volume over 1 year in a group of multiple sclerosis (MS) patients on dimethyl fumarate (DMF) therapy (blue) in comparison to patients on no disease-modifying therapy (noDMT) (red). Means and standard deviations are shown. Total DGM $=$ thalamus + caudate + putamen + globus pallidus. Exact Wilcoxon tests indicated that the putamen volume change differed significantly between the two groups, showing a lower rate of atrophy in the DMF group $(p=0.02) ; p$ values were calculated with time between scans as a covariate; ${ }^{*} p<0.05$ 
results were found when adjusting for scan interval (both $p>0.30$, van Elteren test). T2LV increased by $0.47 \pm 1.25 \mathrm{ml}$ in the DMF group and $0.05 \pm 0.47$ in the noDMT group. The number of Gad-enhancing lesions decreased by $0.25 \pm 1.25$ in the DMF group and $0.13 \pm 0.35$ in the noDMT group.

\section{Additional Statistical Analysis of Treatment Effect Adjusting for Baseline Characteristics}

The results did not change appreciably in multiple variable models when controlling for baseline T2LV, number of Gad-enhancing lesions, and age (in addition to the time between scans). The group comparison of on-study PBVC and putamen volume change remained significant in all models $(p<0.05)$. In subset analyses that accounted for the time between scans and were also balanced on age ( $<52$ years, $n=22)$, T2LV $(<7.0 \mathrm{ml}, n=20)$, number of Gad-enhancing lesions $(\leq 1$, $n=23)$, and with all three restrictions $(n=17)$, the group differences in the changes in putamen volume and PBVC remained meaningful in favor of the DMF group. For example, in subset analyses $(n=17)$ with concomitant reduced power, where the two groups were balanced on all three restrictions, the predicted on-study putamen volume decrease was $0.07 \mathrm{ml}$ for DMF and $0.31 \mathrm{ml}$ for noDMT $(p=0.10)$; the predicted PBVC decrease was 0.33 for DMF and 1.05 for noDMT $(p=0.02)$.

\section{On-Study Clinical Changes}

When comparing DMF and noDMT groups in terms of on-study clinical relapses, no significant differences were noted; one patient in the noDMT group experienced a relapse (per patient relapse rate $12.5 \%$ ) in comparison to 2 patients in the DMF group (rate 10\%) $(p=1.0)$. In addition, no significant differences were found between groups in terms of on-study changes in EDSS score (DMF group: $0 \pm 1.19$ vs. noDMT group: $-0.56 \pm 1.05, p=0.22$ ) or T25FW (DMF group: $0.12 \pm 0.85 \mathrm{~s}$ vs. noDMT: $0.36 \pm 1.36 \mathrm{~s}, p=0.82$ ). Similar results were found when adjusting for time between baseline and follow-up examination $(p=0.23$ and $p=0.43$ for on-study changes in EDSS score and $\mathrm{T} 25 \mathrm{FW}$, respectively).

\section{Correlation Analysis}

When assessing relationships between DGM volume and lesions in all subjects, a significant correlation was found between total DGM volume and $\mathrm{T} 2 \mathrm{LV}$ at baseline (Spearman $r=-0.45, p=0.02$ ); however, no significant correlations were found between the on-study changes in total DGM volume and on-study changes in T2LV, baseline T2LV, or baseline age $(p>0.35)$. In addition, no significant correlations were found between baseline DGM volume and T25FW or EDSS score (all $p>0.05$; baseline total DGM volume vs. baseline EDSS showed a trend toward significance: $r=-0.32, p=0.09$ ). Similarly, no significant correlations were detected between baseline T2LV and baseline T25FW or EDSS score (both $p>0.85$ ). Regarding the ability of baseline variables to predict on-study change in whole brain volume, no significant correlations were detected when comparing baseline T2LV or age to PBVC (all $p>0.80$ ).

\section{DISCUSSION}

The purpose of this pilot study was to compare the rate of cerebral GM atrophy in patients with RRMS recently started on DMF vs. untreated 
patients. Our results show that the DMF group experienced a lower rate of whole brain atrophy than the noDMT group. Additionally, the DMF group had a lower rate of putamen atrophy. No significant on-study differences were detected between groups in caudate, globus pallidus, thalamus, total DGM volume, T2 lesion volume, or clinical measures. These exploratory results provide novel information both in the suggestion of a treatment effect on brain atrophy in the first year after starting DMF and in the demonstration of such effects on GM atrophy. However, these findings should be considered preliminary due to the small sample size and the non-randomized study design.

The results of the present study suggest a treatment effect on brain atrophy appearing in the first year after the start of DMF therapy. In the two pivotal phase III RRMS trials of DMF, a dosage of $240 \mathrm{mg}$ twice a day showed a partial but significant effect at 2 years in limiting the rate of whole brain atrophy compared to placebo. In the DEFINE study, DMF showed a statistically significant $21 \%$ lower rate of atrophy from 0 to 24 months, and a significant 30\% lower rate from 6 to 24 months of the study; however, a 1-year time point MRI scan was not performed [37]. In the CONFIRM study, DMF showed a statistically significant 32\% lower rate of atrophy between years 1 and 2 , although no significant treatment effect was detected in the first year [38]. With regard to the other approved DMTs for RRMS with effects on limiting brain atrophy apparent in the first year, fingolimod has shown an effect on whole brain atrophy in two pivotal phase III studies, apparent at 6 months, as compared to placebo [43, 44]. Patients on teriflunomide experienced significant reductions in brain volume change in comparison to placebo at 1 and 2 years [42]. Additionally, one of the pivotal phase III studies of alemtuzumab showed a lower rate of whole brain atrophy in comparison to patients on IFN $\beta-1$ a at 1 year [58]; the treatment effect was also apparent over 2 years [40, 41]. Similarly, patients on daclizumab experienced significant reductions in the rate of whole brain volume loss compared to patients on IFN $\beta-1 \mathrm{a}$ in both the first and second year of treatment [59]. The demonstration of such early treatment effects on brain atrophy has otherwise typically required 2 years in MS clinical trials $[13,19,34]$. For instance, patients on intramuscular or subcutaneous IFN $\beta-1 \mathrm{a}$ showed a significant reduction in whole brain atrophy in comparison to patients on placebo after 2 years, but not in the first year $[6,35]$. Likewise, patients on GA demonstrated a significant reduction in the rate of whole brain volume loss compared to placebo only in the second 9 months after the start of therapy [36]. Patients on natalizumab showed a significant reduction in the rate of whole brain atrophy compared to placebo in the second but not first year of therapy [39]. The lack of an early treatment effect in these studies has been attributed to two main factors. First, pseudoatrophy may occur in the initial few months after starting DMTs, due to a net loss of edema fluid and inflammatory cells from the brain to the systemic circulation [13, 21, 34]. Second, a time delay may exist for some DMTs, for which the downstream results of their immunomodulatory benefits on ultimate tissue loss may require several months to achieve [6].

To further understand the effect of DMF on brain atrophy, the present study focused on its impact on GM atrophy. Analysis of DGM structures showed a lower rate of atrophy of the putamen over 1 year in the DMF vs. the noDMT group. A limited number of previous 
studies have assessed the impact of other DMTs on cerebral GM $[19,60]$. For instance, studies have shown that the effect of IFN $\beta$ - 1 a on brain atrophy may be driven primarily by its impact on GM atrophy [23, 45, 46]. In addition, emerging studies have suggested that natalizumab limits cerebral GM atrophy or other structural changes [12, 47]. A treatment effect on GM atrophy would have potentially high relevance for patients. GM atrophy occurs early in MS, is clinically relevant, and worsens as the disease progresses $[1,5,7,10,13,15,17,18,24-33,57,61,62]$. Atrophy in the DGM can be detected during the first clinical stages of MS, even as early as the first few years after symptom onset [26].

There are several possible mechanisms by which the MS disease process leads to DGM atrophy. Considering these will help us understand how DMF may exert its therapeutic effect. First, the DGM is highly interconnected with the rest of the brain [63-65], and thus is quite vulnerable to the "dying back" (Wallerian degeneration) effects of neuronal loss following MS-related WM demyelination and axonal transection [65-68]. This would be consistent with the present findings, showing a significant inverse correlation between T2LV and total DGM volume. Second, the DGM is thought to be a site of excessive iron deposition in patients with MS, which has been linked to physical disability, cognitive dysfunction, and brain atrophy $[69,70]$. While deposition of iron may represent purely an epiphenomena of neurodegeneration, one must consider the possibility that iron-mediated (or other forms of) oxidative stress and lipid peroxidation may target the DGM [71]. The third aspect to consider is direct injury to the DGM by the presence of demyelinating foci (lesions); 7T MRI has been pivotal in showing an abundance of such lesions, which are linked to physical disability, progressive disease, and cortical lesions [72]. These are histologically characterized by demyelination, inflammation, and axonal damage [64]. The fourth theory is based on the proximity of the DGM to the ventricular CSF, in which activated lymphocytes are thought to enter via the choroid plexus [73]. DMF has the potential to confer its protective effect on brain atrophy by targeting all four of these proposed mechanisms. This includes its neuroprotective effects via the Nrf2 antioxidant pathway [48], and a wide range of immune effects, such as decreasing T-cells [74], increasing the number of circulating regulatory B-cells [75], exerting immunomodulatory effects on T-cell subsets and antigen-presenting cells [76], downregulating pro-inflammatory cytokines [77], and upregulating the anti-inflammatory cytokine interleukin 10 [78].

Our study has several potential limitations and internal inconsistencies that must be considered. For example, the present study did not show a treatment effect on MRI-defined lesions. This contradicts the findings of phase III clinical trials that have indicated the ability of DMF to limit the appearance of new or enlarging $\mathrm{T} 2$ and Gad lesions [37, 38]. Additionally, we noted a lack of treatment effect on clinical measures including relapse rate, EDSS score, and T25FW. Such inconsistencies may be a result of the small sample size, non-randomized comparison, and the limited duration of the study, given that previous trials assessed treatment effects over 2 years as opposed to 1 year. Our results demonstrated a significant effect on putamen volume change, but not on any other DGM structure or total DGM. This may be due to the fact that DGM nuclei are small structures with complex shapes and sizes, making them 
susceptible to measurement biases. It may also suggest that a longer treatment period would yield volume changes more readily detected by our methods of quantification. In addition, the patients in the DMF arm had been previously treated with other DMTs, and it is not known whether long-term effects of the previous agents influenced our results, even after these treatments were stopped. Lastly, the retrospective design of the present study may have had an impact on our findings. Despite these factors, however, our findings could serve as an impetus for future studies to assess the effects of DMF treatment on cerebral GM in larger prospective controlled studies.

\section{CONCLUSIONS}

The results of this study suggest a treatment effect of DMF on GM atrophy appearing 1 year after starting therapy. However, due to the retrospective study design and sample size, these findings should be considered preliminary, and require confirmation in future investigations.

\section{ACKNOWLEDGEMENTS}

Sponsorship and article processing charges for this study were funded by Biogen. Support for this study was provided through an investigator-initiated trial grant from Biogen, whose employee Lily Lee provided a medical accuracy review of this manuscript. The authors produced the manuscript independently of the funder, and maintained full control of the content. All named authors meet the International Committee of Medical Journal Editors (ICMJE) criteria for authorship for this manuscript, take responsibility for the integrity of the work as a whole, and have given final approval of the version to be published. This work was presented in preliminary form at the 2016 annual meeting of the European Committee on Treatment and Research in Multiple Sclerosis (ECTRIMS), London, UK.

Disclosures. R. Bakshi has received consulting fees from EMD Serono, Genentech, Sanofi-Genzyme, and Novartis, and research support from Biogen, EMD-Serono, Novartis, and Sanofi-Genzyme. S.L. Dupuy, S. Tauhid, S. Hurwitz, R. Chu, and F. Yousuf have nothing to disclose.

Compliance with Ethics Guidelines. All procedures followed were in accordance with the ethical standards of the responsible committee on human experimentation (institutional and national) and with the Helsinki Declaration of 1964, as revised in 2013. Informed consent was not required due to the retrospective nature of the study.

Open Access. This article is distributed under the terms of the Creative Commons Attribution-NonCommercial 4.0 International License (http://creativecommons.org/licenses/ by-nc/4.0/), which permits any noncommercial use, distribution, and reproduction in any medium, provided you give appropriate credit to the original author(s) and the source, provide a link to the Creative Commons license, and indicate if changes were made.

\section{REFERENCES}

1. Bakshi R, Dandamudi VS, Neema M, De C, Bermel RA. Measurement of brain and spinal cord atrophy by magnetic resonance imaging as a tool to monitor multiple sclerosis. J Neuroimaging. 2005;15: 30S-45S.

2. Filippi M, Wolinsky JS, Comi G. CORAL Study Group. Effects of oral glatiramer acetate on clinical and MRI-monitored disease activity in patients with 
relapsing multiple sclerosis: a multicentre, double-blind, randomised, placebo-controlled study. Lancet Neurol. 2006;5:213-20.

3. Filippi M, Rocca MA, Arnold DL, et al. EFNS guidelines on the use of neuroimaging in the management of multiple sclerosis. Eur J Neurol. 2006;13:313-25.

4. Zivadinov R, Bakshi R. Role of MRI in multiple sclerosis I: inflammation and lesions. Front Biosci. 2004;9:665-83.

5. Filippi M, Preziosa P, Rocca MA. Magnetic resonance outcome measures in multiple sclerosis trials: time to rethink? Curr Opin Neurol. 2014;27:290-9.

6. Rudick RA, Fisher E, Lee JC, Simon J, Jacobs L. Use of the brain parenchymal fraction to measure whole brain atrophy in relapsing-remitting MS. Multiple Sclerosis Collaborative Research Group. Neurology. 1999;53:1698-704.

7. Sharma J, Sanfilipo MP, Benedict RH, Weinstock-Guttman B, Munschauer FE 3rd, Bakshi R. Whole-brain atrophy in multiple sclerosis measured by automated versus semiautomated MR imaging segmentation. AJNR Am J Neuroradiol. 2004;25:985-96.

8. Bakshi R, Yeste A, Patel B, et al. Serum lipid antibodies are associated with cerebral tissue damage in multiple sclerosis. Neurol Neuroimmunol Neuroinflamm. 2016;3:e200.

9. Chu R, Hurwitz S, Tauhid S, Bakshi R. Deep gray matter segmentation from 1.5T vs. 3T MRI in normal controls and patients with multiple sclerosis meeting of the American Academy of Neurology, Vancouver, Canada. Neurology. 2016;86(P4):171.

10. Chu R, Tauhid S, Glanz B, et al. Whole brain volume measured from $1.5 \mathrm{~T}$ versus $3 \mathrm{~T}$ MRI in healthy subjects and patients with multiple sclerosis. J Neuroimaging. 2016;26:62-7.

11. Dell'Oglio E, Ceccarelli A, Glanz BI, et al. Quantification of global cerebral atrophy in multiple sclerosis from 3T MRI using SPM: the role of misclassification errors. J Neuroimaging. 2015;25:191-9.

12. Khalid F, Tauhid S, Chua AS, et al. A longitudinal uncontrolled study of cerebral gray matter volume in patients receiving natalizumab for multiple sclerosis. Int J Neurosci. 2016 [Epub ahead of print].

13. Bermel RA, Bakshi R. The measurement and clinical relevance of brain atrophy in multiple sclerosis. Lancet Neurol. 2006;5:158-70.
14. Durand-Dubief F, Belaroussi B, Armspach JP, et al. Reliability of longitudinal brain volume loss measurements between 2 sites in patients with multiple sclerosis: comparison of 7 quantification techniques. AJNR Am J Neuroradiol. 2012;33:1918-24.

15. Maghzi AH, Revirajan N, Julian LJ, et al. Magnetic resonance imaging correlates of clinical outcomes in early multiple sclerosis. Mult Scler Relat Disord. 2014;3:720-7.

16. Tauhid S, Neema M, Healy BC, Weiner H, Bakshi R. MRI phenotypes based on cerebral lesions and atrophy in patients with multiple sclerosis. J Neurol Sci. 2014;346:250-4.

17. Zivadinov $\mathrm{R}$, Uher $\mathrm{T}$, Hagemeier $\mathrm{J}$, et al. A serial 10-year follow-up study of brain atrophy and disability progression in RRMS patients. Mult Scler. 2016 [Epub ahead of print].

18. Rojas JI, Patrucco L, Miguez J, Cristiano E. Brain atrophy in multiple sclerosis: therapeutic, cognitive and clinical impact. Arg Neuropsiquiatr. 2016;74:235-43.

19. Vidal-Jordana A, Sastre-Garriga J, Rovira A, Montalban X. Treating relapsing-remitting multiple sclerosis: therapy effects on brain atrophy. J Neurol. 2015;262:2617-26.

20. Stankiewicz JM, Glanz BI, Healy BC, et al. Brain MRI lesion load at $1.5 \mathrm{~T}$ and $3 \mathrm{~T}$ versus clinical status in multiple sclerosis. J Neuroimaging. 2011;21:e50-6.

21. Khoury S, Bakshi R. Cerebral pseudoatrophy or real atrophy after therapy in multiple sclerosis. Ann Neurol. 2010;68:778-9.

22. De Stefano N, Arnold DL. Towards a better understanding of pseudoatrophy in the brain of multiple sclerosis patients. Mult Scler. 2015;21:675-6.

23. Fisher E, Nakamura K, Lee JC, You X, Sperling B, Rudick RA. Effect of intramuscular interferon beta-1a on gray matter atrophy in relapsing-remitting multiple sclerosis: a retrospective analysis. Mult Scler. 2016;22:668-76.

24. Dalton CM, Chard DT, Davies GR, et al. Early development of multiple sclerosis is associated with progressive grey matter atrophy in patients presenting with clinically isolated syndromes. Brain. 2004;127:1101-7.

25. Bakshi R, Neema M, Tauhid S, et al. An expanded composite scale of MRI-defined disease severity in multiple sclerosis: MRDSS2. Neuro Report. 2014;25:1156-61. 
26. Bergsland N, Horakova D, Dwyer MG, et al. Subcortical and cortical gray matter atrophy in a large sample of patients with clinically isolated syndrome and early relapsing-remitting multiple sclerosis. AJNR Am J Neuroradiol. 2012;33:1573-8.

27. Miller DH, Barkhof F, Frank JA, Parker GJ, Thompson AJ. Measurement of atrophy in multiple sclerosis: pathological basis, methodological aspects and clinical relevance. Brain. 2002;125:1676-95.

28. Modica CM, Bergsland N, Dwyer MG, et al. Cognitive reserve moderates the impact of subcortical gray matter atrophy on neuropsychological status in multiple sclerosis. Mult Scler. 2016;1:36-42.

29. Pagani E, Rocca MA, Gallo A, et al. Regional brain atrophy evolves differently in patients with multiple sclerosis according to clinical phenotype. AJNR Am J Neuroradiol. 2005;26:341-6.

30. Ruggieri S, Petracca M, Miller A, et al. Association of deep gray matter damage with cortical and spinal cord degeneration in primary progressive multiple sclerosis. JAMA Neurol. 2015;72:1466-74.

31. Steckova T, Hlustik P, Sladkova V, Odstrcil F, Mares J, Kanovsky P. Thalamic atrophy and cognitive impairment in clinically isolated syndrome and multiple sclerosis. J Neurol Sci. 2014;342:62-8.

32. Janardhan V, Bakshi R. Quality of life and its relationship to brain lesions and atrophy on magnetic resonance images in 60 patients with multiple sclerosis. Arch Neurol. 2000;57:1485-91.

33. Bakshi R, Czarnecki D, Shaikh ZA, et al. Brain MRI lesions and atrophy are related to depression in multiple sclerosis. Neuro Report. 2000;11:1153-8.

34. Zivadinov R, Reder AT, Filippi M, et al. Mechanisms of action of disease-modifying agents and brain volume changes in multiple sclerosis. Neurology. 2008;71:136-44.

35. Filippi $\mathbf{M}$, Rovaris $M$, Inglese $M$, et al. Interferon beta-1a for brain tissue loss in patients at presentation with syndromes suggestive of multiple sclerosis: a randomised, double-blind, placebo-controlled trial. Lancet. 2004;364:1489-96.

36. Sormani MP, Rovaris M, Valsasina P, Wolinsky JS, Comi G, Filippi M. Measurement error of two different techniques for brain atrophy assessment in multiple sclerosis. Neurology. 2004;62:1432-4.

37. Arnold DL, Gold R, Kappos L, et al. Effects of delayed-release dimethyl fumarate on MRI measures in the phase 3 DEFINE study. J Neurol. 2014;261:1794-802.
38. Miller DH, Fox RJ, Phillips JT, et al. Effects of delayed-release dimethyl fumarate on MRI measures in the phase 3 CONFIRM study. Neurology. 2015;84:1145-52.

39. Miller DH, Soon D, Fernando KT, et al. MRI outcomes in a placebo-controlled trial of natalizumab in relapsing MS.

Neurology. 2007;68:1390-401.

40. Cohen JA, Coles AJ, Arnold DL, et al. Alemtuzumab versus interferon beta $1 \mathrm{a}$ as first-line treatment for patients with relapsing-remitting multiple sclerosis: a randomised controlled phase 3 trial. Lancet. 2012;380:1819-28.

41. Coles AJ, Twyman CL, Arnold DL, et al. Alemtuzumab for patients with relapsing multiple sclerosis after disease-modifying therapy: a randomised controlled phase 3 trial. Lancet. 2012;380:1829-39.

42. Radue EW, Sprenger $T$, Gaetano $L$, et al. Teriflunomide slows brain volume loss in relapsing MS: a SIENA analysis of the TEMSO MRI dataset. 2016 meeting of the American Academy of Neurology, Vancouver, Canada. Neurology. 2016:P3.089.

43. Calabresi PA, Radue EW, Goodin D, et al. Safety and efficacy of fingolimod in patients with relapsing-remitting multiple sclerosis (FREEDOMS II): a double-blind, randomised, placebo-controlled, phase 3-trial. Lancet Neurol. 2014; 13:545-56.

44. Kappos L, Radue EW, O'Connor P, et al. A placebo-controlled trial of oral fingolimod in relapsing multiple sclerosis. $\mathrm{N}$ Engl $\mathrm{J}$ Med. 2010;362:387-401.

45. Zivadinov $\mathrm{R}$, Locatelli $\mathrm{L}$, Cookfair $\mathrm{D}$, et al. Interferon beta-1a slows progression of brain atrophy in relapsing-remitting multiple sclerosis predominantly by reducing gray matter atrophy. Mult Scler. 2007;13:490-501.

46. Benfeldt $\mathrm{K}$, Egger $\mathrm{H}$, Nichols TE, et al. Effect of immunomodulatory medication on regional gray matter loss in relapsing-remitting multiple sclerosis-a longitudinal MRI study. Brain Res. 2010;1325:174-82.

47. Romme Christensen J, Ratzer R, Bornsen L, et al. Natalizumab in progressive MS: results of an open-label, phase 2A, proof-of-concept trial. Neurology. 2014;82:1499-507.

48. Linker RA, Lee DH, Ryan S, et al. Fumaric acid esters exert neuroprotective effects in neuroinflammation via activation of the Nrf2 antioxidant pathway. Brain. 2011;134:678-92. 
49. Scannevin RH, Chollate S, Jung MY, et al. Fumarates promote cytoprotection of central nervous system cells against oxidative stress via the nuclear factor (erythroid-derived 2)-like 2 pathway. J Pharmacol Exp Ther. 2012;341:274-84.

50. Fox RJ, Miller DH, Phillips JT, et al. Placebo-controlled phase 3 study of oral BG-12 or glatiramer in multiple sclerosis. $\mathrm{N}$ Engl J Med. 2012;367:1087-97.

51. Gold R, Kappos L, Arnold DL, et al. Placebo-controlled phase 3 study of oral BG-12 for relapsing multiple sclerosis. $\mathrm{N}$ Engl $\mathrm{J}$ Med. 2012;367:1098-107.

52. Kappos L, Gold R, Miller DH, et al. Efficacy and safety of oral fumarate in patients with relapsing-remitting multiple sclerosis: a multicentre, randomized, double-blind, placebo-controlled phase IIb study. Lancet. 2008;372:1463-72.

53. Gold R, Arnold DL, Bar-Or A, et al. Long-term effects of delayed-release dimethyl fumarate in multiple sclerosis: Interim analysis of ENDORSE, a randomized extension study. Mult Scler. 2016 [Epub ahead of print].

54. Viglietta V, Miller D, Bar-Or A, et al. Efficacy of delayed-release dimethyl fumarate in relapsing-remitting multiple sclerosis: integrated analysis of the phase 3 trials. Ann Clin Transl Neurol. 2015;2:103-18.

55. Smith SM, De Stefano N, Jenkinson M, Matthews PM. Normalized accurate measurement of longitudinal brain change. J Comput Assist Tomogr. 2001;25:466-75.

56. Patenaude B, Smith SM, Kennedy DN, Jenkinson M. A Bayesian model of shape and appearance for subcortical brain segmentation. Neuroimage. 2011;56:907-22.

57. Bermel RA, Sharma J, Tjoa CW, Puli SR, Bakshi R. A semiautomated measure of whole-brain atrophy in multiple sclerosis. J Neurol Sci. 2003;208:57-65.

58. Schippling S, Cohen J, Coles A, et al. Alemtuzumab-treated patients with RRMS demonstrate durable slowing of brain volume loss over 5 years despite most being treatment-free for 4 years: CARE-MS I and II extension study. American Academy of Neurology, Vancouver, Canada. Neurology. 2016:S51.001.

59. Rose J, Wiendl H, Arnold DL, et al. Daclizumab HYP reduced brain MRI lesion activity compared with interferon beta-1a: Results from DECIDE study. 2015 meeting of the American Academy of
Neurology, Washington, D.C., USA. Neurology. 2015:P7.252.

60. Calabrese M, Gajofatto A, Benedetti MD. Therapeutic strategies for relapsing-remitting multiple sclerosis: a special focus on reduction of grey mater damage as measured by brain atrophy. Expert Rev Neurother. 2014;14:1417-28.

61. Houtchens MK, Benedict RH, Killiany R, et al. Thalamic atrophy and cognition in multiple sclerosis. Neurology. 2007;69:1213-23.

62. Pirko I, Lucchinetti CF, Sriram S, Bakshi R. Gray matter involvement in multiple sclerosis. Neurology. 2007;68:634-42.

63. Bakshi R, Miletich RS, Kinkel PR, Emmet ML, Kinkel WR. High-resolution fluorodeoxyglucose positron emission tomography shows both global and regional cerebral hypometabolism in multiple sclerosis. J Neuroimaging. 1998;8:228-34.

64. Vercellino M, Masera S, Lorenzatti $M$, et al. Demyelination, inflammation, and neurodegeneration in multiple sclerosis deep gray matter. J Neuropathol Exp Neurol. 2009;68:489-502.

65. Kipp M, Wagenknecht N, Beyer C, Samer S, Wuerfel $J$, Nikoubashman $O$. Thalamus pathology in multiple sclerosis: from biology to clinical application. Cell Mol Life Sci. 2015;72:1127-47.

66. Ciccarelli O, Barkhof F, Bodini B, et al. Pathogenesis of multiple sclerosis: insights from molecular and metabolic imaging. Lancet Neurol. 2014;13:807-22.

67. Prinster A, Quarantelli M, Orefice G, et al. Grey matter loss in relapsing-remitting multiple sclerosis: a voxel-based morphometry study. Neuroimage. 2006;29:859-67.

68. Henry RG, Shieh M, Amirbekian B, Chung S, Okuda DT, Pelletier D. Connecting white matter injury and thalamic atrophy in clinically isolated syndromes. J Neurol Sci. 2009;282:61-6.

69. Stankiewicz J, Panter SS, Neema M, Arora A, Batt $\mathrm{CE}$, Bakshi R. Iron in chronic brain disorders: imaging and neurotherapeutic implications. Neurotherapeutics. 2007;4:371-86.

70. Brass SD, Chen NK, Mulkern RV, Bakshi R. Magnetic resonance imaging of iron deposition in neurological disorders. Top Magn Reson Imaging. 2006;17:31-40.

71. Haider L. Inflammation, iron, energy failure, and oxidative stress in the pathogenesis of multiple sclerosis. Oxid Med Cell Longev. 2015; doi:10. 1155/2015/725370. 
72. Harrison DM, Oh J, Roy S, et al. Thalamic lesions in multiple sclerosis by 7T MRI: clinical implications and relationship to cortical pathology. Mult Scler. 2015;21:1139-50.

73. Kivisäkk P, Imitola J, Rasmussen S, et al. Localizing central nervous system immune surveillance: meningeal antigen-presenting cells activate $\mathrm{T}$ cells during experimental autoimmune encephalomyelitis. Ann Neurol. 2009;65:457-69.

74. Spencer CM, Crabtree-Hartman EC, Lehmann-Horn $\mathrm{K}$, Cree BA, Zamvil SS. Reduction of CD8(+) T lymphocytes in multiple sclerosis patients treated with dimethyl fumarate. Neurol Neuroimmunol Neuroinflamm. 2015;2:e76.

75. Linker RA, Haghikia A. Dimethyl fumarate in multiple sclerosis: latest developments, evidence and place in therapy. Ther Adv Chronic Dis. 2016;7:198-207.

76. Linker RA, Lee DH, Stangel M, Gold R. Fumarates for the treatment of multiple sclerosis: potential mechanisms of action and clinical studies. Expert Rev Neurother. 2008;8:1683-90.

77. Vandermeeren M, Janssens S, Borgers M, Geysen J. Dimethylfumarate is an inhibitor of cytokine-induced E-selectin, VCAM-1, and ICAM-1 expression in human endothelial cells. Biochem Biophys Res Commun. 1997;234:19-23.

78. Schilling S, Goelz S, Linker R, Luehder F, Gold R. Fumaric acid esters are effective in chronic experimental autoimmune encephalomyelitis and suppress macrophage infiltration. Clin Exp Immunol. 2006;145:101-7. 